\title{
Bimanual interference in children performing a dual motor task
}

Citation for published version (APA):

Otte, E., \& van Mier, H. I. (2006). Bimanual interference in children performing a dual motor task. Human Movement Science, 25, 678-693. https://doi.org/10.1016/j.humov.2006.07.008

Document status and date:

Published: 01/01/2006

DOI:

10.1016/j.humov.2006.07.008

Document Version:

Publisher's PDF, also known as Version of record

Document license:

Taverne

Please check the document version of this publication:

- A submitted manuscript is the version of the article upon submission and before peer-review. There can be important differences between the submitted version and the official published version of record.

People interested in the research are advised to contact the author for the final version of the publication, or visit the DOI to the publisher's website.

- The final author version and the galley proof are versions of the publication after peer review.

- The final published version features the final layout of the paper including the volume, issue and page numbers.

Link to publication

\footnotetext{
General rights rights.

- You may freely distribute the URL identifying the publication in the public portal. please follow below link for the End User Agreement:

www.umlib.nl/taverne-license

Take down policy

If you believe that this document breaches copyright please contact us at:

repository@maastrichtuniversity.nl

providing details and we will investigate your claim.
}

Copyright and moral rights for the publications made accessible in the public portal are retained by the authors and/or other copyright owners and it is a condition of accessing publications that users recognise and abide by the legal requirements associated with these

- Users may download and print one copy of any publication from the public portal for the purpose of private study or research.

- You may not further distribute the material or use it for any profit-making activity or commercial gain

If the publication is distributed under the terms of Article $25 \mathrm{fa}$ of the Dutch Copyright Act, indicated by the "Taverne" license above, 


\title{
Bimanual interference in children performing a dual motor task
}

\author{
E. Otte ${ }^{\text {a,b,* }}$, H.I. van Mier ${ }^{\text {a }}$ \\ ${ }^{a}$ Faculty of Psychology, University of Maastricht, P.O. Box 616, 6200 MD Maastricht, The Netherlands \\ ${ }^{\mathrm{b}}$ International Graduate School of Neuroscience, Institute of Neuroinformatics, Ruhr Universität Bochum, \\ D-44780 Bochum, Germany
}

Available online 2 October 2006

\begin{abstract}
The present study addressed the development of bimanual interference in children performing a dual motor task, in which each hand executes a different task simultaneously. Forty right-handed children (aged 4, 5-6, 7-8 and 9-11 years, ten in each age group) were asked to perform a bimanual task in which they had to tap with a pen using the non-preferred hand and simultaneously trace a circle or a square with a pen using the preferred hand as quickly as possible. Tapping and tracing were also performed unimanually. Differences between unimanual and bimanual performance were assessed for number of taps, length of tap trace and mean tracing velocity. It was assumed that with increasing age, better bimanual coordination would result in better performance on the dual task showing less intermanual interference. The results showed that tapping and tracing performance increased with age, unimanually as well as bimanually, consistent with developmental advancement. However, the percentage of intermanual interference due to bimanual performance was not significantly different in the four age groups. Although performing the dual task resulted in mutual intermanual interference, all groups showed a significant effect of tracing shape. More specifically, all age groups showed a larger percentage decrease in tracing velocity when performing the circle compared to the square in the dual task. The present study reveals that children as young as four years are able to coordinate both hands when tapping and tracing bimanually.

(c) 2006 Elsevier B.V. All rights reserved.
\end{abstract}

\footnotetext{
* Corresponding author. Address: IGSN, Institute of Neuroinformatics, Ruhr Universität Bochum, D-44780 Bochum, Germany.

E-mail address: ellen.otte@rub.de (E. Otte).
} 


\section{Introduction}

In everyday life, we constantly perform activities that require the use of our hands. Some movements involve only one hand (unimanual movements) while others require both hands (bimanual movements). In general, it is easier for people to perform unimanual actions than bimanual actions (Annett, 1970; Kelso, Southard, \& Goodman, 1979; Wyke, $1968,1969,1971)$. Nevertheless, some movements require the use of two hands. Eating with knife and fork, opening a jar, clapping your hands or playing the piano are some examples of the great diversity of bimanual movements we use in daily life.

Bimanual movements can be defined in three broad classes (Fagard, 1991). The first, symmetrical complementary movements are the easiest: both hands contribute to a single goal with a similar output. This kind of task requires both hands to perform a different movement but to exert it on the same object, as in clapping one's hands (Wiesendanger, Wicki, \& Rouiller, 1994). This level of bimanual coordination can already be observed early in life, during the first year, when infants reach for large objects (Fagard \& Jacquet, 1989; Goldfield \& Michel, 1986a, 1986b; Michel, Ovrut, \& Harkins, 1985). Asymmetrical complementary movements, or role-differentiated movements (Kimmerle, Mick, \& Michel, 1995; Ramsey \& Weber, 1986) form the second class: both hands contribute to a single goal but with an asymmetrical output. For example, one hand has a supporting or stabilizing function while the other hand manipulates or explores the object, as when opening a jar. While Kimmerle et al. (1995) observed asymmetrical movements in children as young as 7 months, they are more commonly developed at the end of the first year (Fagard \& Marks, 2000; Ramsey, Campos, \& Fenson, 1979), with asymmetrical movements for certain objects not appearing until around 24 months of age (Fagard \& Jacquet, 1989; Ramsey \& Weber, 1986). In general, asymmetrical patterns are more complex, more variable and appear later in life than symmetrical patterns (Fagard \& Jacquet, 1989; Whitall, 1989). The last class of bimanual movements, also the most difficult, consists of dual task movements, in which a person executes an independent movement with each hand simultaneously (Lee, Swinnen, \& Verscheuren, 1995). In this case, two simultaneous but independent bilateral manual activities are required, for example, tapping with one hand while drawing a circle or square simultaneously using the other hand, as required in the current study. While complementary movements have been addressed in developmental research, commonly showing that with increasing age children are better at performing these tasks (Fagard, Hardy-Léger, Kervella, \& Marks, 2001; Fagard \& Jacquet, 1989; Fagard \& Marks, 2000; Kimmerle et al., 1995; Marion, Kilian, Naramor, \& Brown, 2003), dual task movements have mainly been the topic of research in adults (Lee et al., 1995; Serrien, Pogosyan, \& Brown, 2004; Swinnen \& Walter, 1991). The current study was set up to investigate the development of bimanual performance in a dual task in children aged 4-11 years.

When simultaneously performing two independent movements, especially movements with different spatiotemporal patterns, mutual interference is often the result, even though performing each task unimanually poses no problem (Franz, Zelaznik, \& McCabe, 1991; 
Heuer, 1996; Vangheluwe, Puttemans, Wenderoth, Van Baelen, \& Swinnen, 2004; Walter \& Swinnen, 1990, 1992). When different spatiotemporal patterns must be simultaneously produced by contralateral limbs, their trajectories typically reflect characteristics of one another (Kelso, Putnam, \& Goodman, 1983; Konzem, 1987; Swinnen \& Walter, 1988). For example, when performing two independent movements simultaneously, such as rubbing one's stomach and patting one's head, our limbs tend to draw towards one or the other task, producing either rubbing or patting motions with both hands. Results from other bimanual studies have shown interference when participants were asked to tap two different rhythms simultaneously with both hands (Summers, Todd, \& Kim, 1993), or perform an action with one hand while tapping with the other (Klapp, 1979). Even when different bodyparts were used, as in speaking a syllable while tapping (Klapp, 1981) or citing a nursery rhyme and tapping a rhythm (Peters, 1977), interference has been reported. In most cases movements performed with the preferred hand interfere with movements of the nonpreferred hand (Fagard, 1991; Peters, 1981, 1985). On the other hand, mirror movements are often shown to be stronger for the non-preferred than the preferred hand (Armatas, Summers, \& Bradshaw, 1994; Wolff, Gunnoe, \& Cohen, 1983). Additionally, bimanual actions are generally easier if the preferred hand performs the more difficult task (Fowler, Duck, Mosher, \& Mathieson, 1991; Ibbotson \& Morton, 1981; Kelso et al., 1979; Peters, 1985; Todor \& Smiley, 1985; Walter \& Swinnen, 1990). Apparently, it is rather difficult to combine two actions with different temporal structures (Deutsch, 1983; Kelso et al., 1979; Klapp, 1979).

When producing bimanual movements with a different temporal pattern we need to suppress an innate tendency to couple both movements temporally. With effort, this tendency can be overcome and the degree of temporal coupling can be modulated (Cardoso de Oliveira, 2002). Fagard (1991) described a model accounting for temporal differences between the two hands in bimanual movements. This model assumed that each movement is organized in a specific temporal structure; each hand is controlled by a 'central timekeeping process', or 'general movement program' (Schmidt \& Lee, 2005). This general movement program specifies the entire 'shape' of a movement even before it is executed (Cardoso de Oliveira, 2002). Each hand is assumed to have a movement program of its own. When two hands are used at the same time, interference between the hands occurs when the timing, initiation, and temporal parameters for the two hands are different. To perform two independent movements simultaneously, one common motor program has to be established that combines both independent movement programs (Schmidt, Zalaznik, Hawkins, Frank, \& Quinn, 1979). Because temporal aspects are specified by common parameters (Schmidt et al., 1979), movements with a similar timing dynamic should interfere less than movements with a different timing dynamic (Deutsch, 1983; Kelso et al., 1979; Klapp, 1979; Robertson, 2001; Schmidt \& Lee, 2005).

To test the latter, the current study used bimanual movements with different timing dynamics. Children were instructed to trace either a circle or a square using their preferred hand while simultaneously performing a discrete tapping motion using the non-preferred hand. While a circle can be traced continuously, tracing a square will be less continuous or discontinuous, since momentary stops need to be included at the corners. We expected interference between the hands while performing these two independent movements because each movement has a different timing dynamic. More specifically, we anticipated that simultaneously tapping and tracing the circle would be more difficult than simultaneously tapping and tracing the square, because the timing dynamics of the latter are more 
similar than of the former (Deutsch, 1983; Kelso et al., 1979; Klapp, 1979; Robertson, 2001; Schmidt \& Lee, 2005).

Being able to skillfully perform bimanual movements represents a major developmental shift in motor skills and cognitive demands (Bruner, 1970; Connolly \& Dalgleish, 1989; Ramsey \& Weber, 1986; Siegler \& Munakata, 1993). Bimanual movements require intralimb coordination, as well as integration and sequencing of separate motor acts between limbs, i.e., interlimb coordination. It has been suggested that interhemispheric development, more specifically interhemispheric specialization, and transfer play an important role in this process (Andres et al., 1999; Cardoso de Oliveira, 2002; Fagard et al., 2001; Michel et al., 1985; Ramsey et al., 1979). More specifically, performance in bimanual tasks has often been related to the development of the corpus callosum (Berlucchi, Aglioti, Marzi, \& Tassinari, 1995; Kennerley, Diedrichsen, Hazeltine, Semjen, \& Ivry, 2002). Since development of the corpus callosum is thought to be continuing into adolescence (Giedd et al., 1996, 1999; Rauch \& Jinkins, 1994) it might be expected that with increasing age children will perform better at the bimanual dual task resulting in less intermanual interference.

In summary, we expect unimanual task performance to be better than bimanual task performance. More precisely, in the bimanual task we expect less taps, longer tap traces (meaning that taps will not be actual points but rather small lines as a consequence of tracing interference) and a slower tracing velocity. Additionally, we expect to find more interference when tracing the circle than the square in the dual task, because the timing pattern of the latter is more similar to the tapping movements, consequently leading to less interference. Finally, we expect older children to perform better than younger children, and showing less interference in the bimanual task.

\section{Methods}

\subsection{Participants}

A total of 40 normal children participated in the study. Children were divided into four age groups of 10 participants each. Children in the first group were 4 years of age (mean age: 4.6, SD: 0.3). Participants in the second group were 5-6years old (mean age: 5.9, SD: 0.4 ). The third group contained children of 7-8 years (mean age: 7.9 , SD 0.3). The last group consisted of children ranging from 9 to 11 years (mean age: 10.8, SD: 0.5). Children's handedness was assessed using a Dutch version of Annett Handedness Inventory (1967) revised by Briggs and Nebes (1975). We modified this inventory for use by children by replacing two items of the original inventory (i.e., 'striking a match' and 'threading a needle') with items more convenient and familiar for children ('holding a knife when buttering bread' and 'eating with a spoon'). Hand preference for each item was scored on a five-point scale ranging from 'always left' (score -2$)$ to 'always right' $($ score +2$)$. This way scores ranged from -24 (strongly left-handed) to +24 (strongly right-handed). Briggs and Nebes (1975) identified a person as right-handed with a score of +9 or above. Handedness scores showed that all participants were strongly right-handed (mean score for group 1: 18.5, $\mathrm{SD}=4.7$; group 2: 19.1, $\mathrm{SD}=3.8$; group 3: 18.4, $\mathrm{SD}=4.5$; and group 4: 23.0, $\mathrm{SD}=1.9$ ). None of the children had any developmental, neurological, motor or learning problems and all children showed proficient hand use regarding pen movements.

All participants were students of 'AFNORTH International School' and parents or caretakers gave informed consent. The local Ethics Committee approved the study, which 

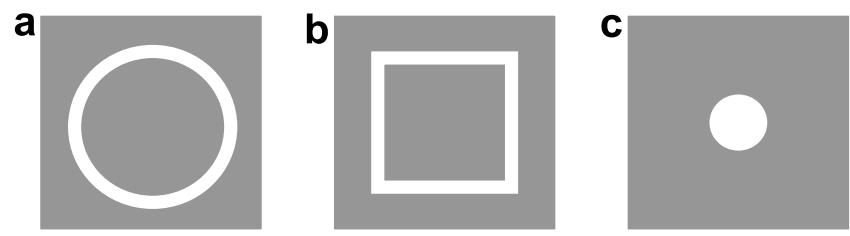

Fig. 1. Cut-out patterns used for circle tracing (a), square tracing (b), and tapping (c).

was carried out according to the ethical guidelines laid down in the Declaration of Helsinki.

\subsection{Material and apparatus}

To ensure that all participants performed the square and circle at the same size two cut out patterns were used; a circle and a square (both with a path length of $26 \mathrm{~cm}$, a track width of $0.5 \mathrm{~cm}$, and a track depth of $0.3 \mathrm{~cm}$ ). To ensure that participants would tap in more or less the same area, a cut out pattern with a diameter of $1.5 \mathrm{~cm}$ was used for tapping. The patterns were cut out of cardboard (see Fig. 1) and were attached on a transparent sheet that was fixed onto the writing tablet. Participants performed the tasks on a Wacom writing tablet, which was connected to a laptop, using two wireless non-inking pens. The data recorded are the $x$ - and $y$-coordinates every $10 \mathrm{~ms}$ using Oasis software (De Jong, Hulstijn, Kosterman, \& Smits-Engelsman, 1996). Data records for all trials were low-pass filtered with a cutoff frequency of $10 \mathrm{~Hz}$ using a second order Butterworth filter. Pen trajectories of tapping and drawing movements of each hand were stored independently, allowing separate analyses for each hand.

\subsection{Task description and procedure}

Parents or caretakers of each child received an informed consent and the handedness questionnaire plus instructions. They were asked to sign the informed consent and to fill out the handedness questionnaire, by having the child perform the actions on the questionnaire and score accordingly. Before the testing procedure started, children were asked to draw a picture. This way, participants got acquainted with the pen and writing tablet while at the same time, the child's preferred hand could be verified with the handedness score. For all children, the score on the handedness test matched the preferred hand in the drawing task.

The children were tested outside of the classroom, in a quiet corner in the hallway. A table and chair with the appropriate size for the child were set up. The instructor sat on the right side of the child to present the unimanual and bimanual tasks. Subsequently the testprocedure was explained and testing started. Participants started with unimanual performance consisting of four unimanual trials, which were performed for a period of $30 \mathrm{~s}$ each. For the first unimanual trial, the child was asked to tap as quickly as possible with the pen in the preferred hand. For the second trial, the same action had to be performed but now with the non-preferred hand. In the third unimanual trial, a circle had to be traced using a pen by following the track of the cut out pattern continuously. The last unimanual trial required the same action to be executed but now a square had to be traced. The circle and square were always traced using the preferred hand. 
Unimanual performance was followed by bimanual performance. Bimanual performance also consisted of four trials, which were performed for a period of $30 \mathrm{~s}$ each. In the first two trials, participants were instructed to tap using the non-preferred hand and at the same time trace the circle or the square using the preferred hand. Half of the participants in each group started tracing the square and the other half started tracing the circle. For the last two trials (three and four), the bimanual tasks of trial 1 and 2 were repeated. So again, participants had to tap with the non-preferred hand while tracing the profile of circle or square with the preferred hand.

Participants were instructed to perform the tasks as fast as possible. A break was included between the unimanual and bimanual session and between trials, if needed. Total duration of the experiment was about 30-40 min. Due to several constraints (attention limitations for the youngest age group and time restrictions for the older children because of classes) we were not able to examine bimanual performance with the reverse task, i.e., tracing using the non-preferred hand and tapping using the preferred hand.

\subsection{Data analysis}

Data records for all trials were low-pass filtered with a cutoff frequency of $10 \mathrm{~Hz}$ using a second order Butterworth filter and the drawing trajectories were displayed on the computer screen. For each trial, we analyzed a total of $20 \mathrm{~s}$ starting at the first pen movement on the writing tablet. Because the younger children did not always start immediately after the go-signal, this way, similar movement durations were measured. The experimenter determined the beginning and end of each trial by means of an interactive computer program. The following dependent variables were calculated for this period using OASIS software (De Jong et al., 1996): the number of taps and tap trace length (the length of the pen trace left on the paper) for the tapping task, and mean tracing velocity for the tracing task. We expected that interference from the tracing movements on the tapping movements would be displayed in longer tap trace lengths in the bimanual task, meaning that taps would not be actual points but rather small lines as a consequence of tracing interference.

The data were analyzed using a split-plot design (between factor: age group; within factors: hand (preferred vs. non-preferred) for tapping, condition (unimanual vs. bimanual) and repetition (trial 1 vs. trial 2 in the bimanual task) for tapping and tracing, as well as shape (circle vs. square) for tracing) with ANOVA repeated measurement testing. When a significant main effect was found for age group, comparisons between age groups were examined post-hoc using a Bonferroni correction.

Additionally, we analyzed the percentage of intermanual interference for each hand by calculating the difference between bimanual and unimanual performance, then dividing by unimanual performance and multiplying by 100. An ANOVA was performed for all dependent variables with age group and shape as independent variables.

\section{Results}

The results will be discussed per effect for each variable separately. When a significant main effect was found for age group, comparisons between age groups were examined post-hoc using a Bonferroni correction. Only significant interactions will be mentioned. 

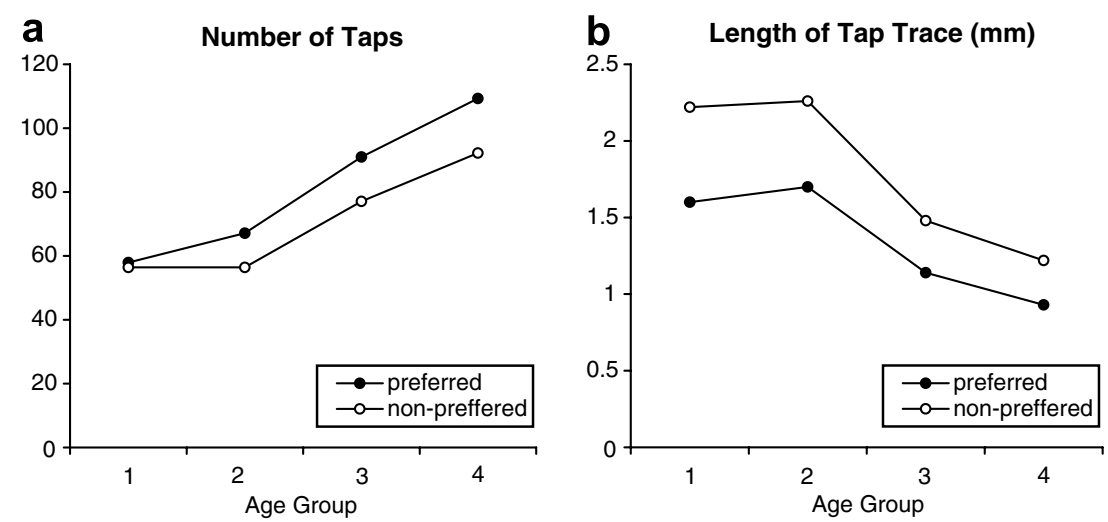

Fig. 2. Effects of age group and hand (preferred vs. non-preferred) for number of taps (a) and length of tap trace (b).

\subsection{Unimanual tapping}

First, we analyzed differences in unimanual tapping performance between the preferred and the non-preferred hand. We found a significant main effect for age group $(F(3,36)=$ $44.34, p<.001 ; F(3,36)=7.03, p<.001$ ), (for number of taps and tap trace length, respectively) and hand (preferred hand vs. non-preferred hand) $(F(1,36)=38.07, p<.001$ for number of taps, and $F(1,36)=9.73, p<.01$ for tap trace length). As shown in Fig. 2, older children tapped significantly more often and with smaller tap trace lengths than younger children did. Tapping with the preferred hand resulted in a mean of 81.3 taps and a mean tap trace of $1.3 \mathrm{~mm}$, while on average 70.5 taps of $1.8 \mathrm{~mm}$ were performed with the non-preferred hand. For number of taps a significant interaction of age group and hand was found $(F(3,36)=3.68, p<.05)$. As can be seen in Fig. 2, differences between the preferred and nonpreferred hand were more pronounced in the older children.

Post-hoc comparisons between the age groups showed that the eldest children (group 4) tapped significantly more often than the other age groups $(p<.001$ for age group 1 and 2 , $p<.01$ for group 3). Group 3 tapped faster than age group 1 and $2(p<.001)$. Regarding tap trace length, post-hoc analyses showed significant differences between age group 4 and age group 1 and $2(p<.01)$, and between age group 3 and $2(p<.05)$.

\subsection{Unimanual tracing}

Unimanual tracing showed a significant main effect for age group $(F(3,36)=31.58$, $p<.001)$ and for shape (circle vs. square) $(F(1,36)=431.89, p<.001)$. Fig. $3 \mathrm{c}$ shows that with increasing age children performed faster on the unimanual-tracing task. Differences between the shapes were measured by comparing participants' performance on the circle vs. their performance on the square. As shown in Fig. 3c, children traced the circle with a speed that was about twice as fast as the square (mean velocity of $32.7 \mathrm{~cm} / \mathrm{s}$ for the circle; $16.2 \mathrm{~cm} / \mathrm{s}$ for the square). There was a significant interaction between age group and shape $(F(3,36)=26.08, p<.001)$, with age differences being more prominent for the circle than the square. 

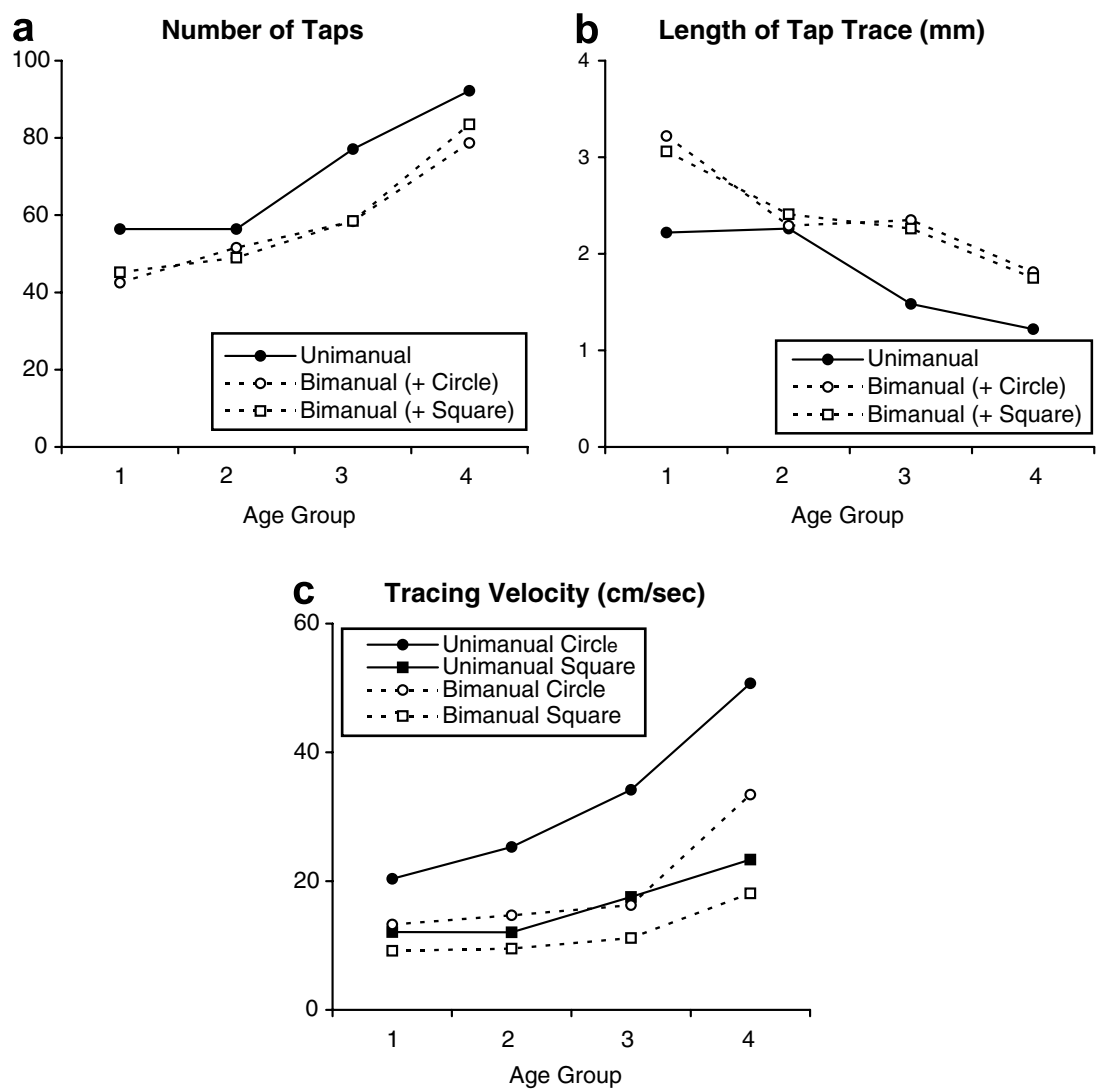

Fig. 3. Effects of age group, condition (unimanual vs. bimanual) and shape (circle vs. square) for number of taps (a), length of tap trace (b) and mean tracing velocity (c). Note that the number of taps and length of tap trace in the bimanual condition are averaged over two trials and are displayed for trials combined with either circle or square tracing.

Post-hoc comparisons for age group showed that the eldest children (group 4) were significantly faster than the other age groups $(p<.001)$. Age group 3 was faster than age group 1 and $2(p<.01$ and $p<.05$, respectively).

\subsection{Unimanual vs. bimanual: Tapping}

Bimanual performance was measured as a comparison between tapping with the nonpreferred hand in the unimanual and bimanual tasks. There was no significant effect between trials 1 and 2 in the bimanual trials regarding the number and trace length of the taps. The effect of shape was also not significant in the bimanual task, meaning that the number and trace length of the taps performed with the non-preferred hand was not significantly different when performing the circle or square with the preferred hand (see Figs. $3 a$ and $b$ ). Therefore, we averaged both trials and shapes in the bimanual task.

We found a significant main effect for age group for number of taps $(F(3,36)=16.08$, $p<.001)$ and for tap trace length $(F(3,36)=7.47, p<.001)$. The developmental effect can be seen in Figs. 3a and b; older children tapped significantly more and with shorter tap trace 
lengths than the younger ones in both unimanual and bimanual trials. The effect of condition was also significant for number of taps $(F(1,36)=21.51, p<.001)$ and tap trace length $(F(1,36)=11.16, p<.01)$. The number of taps decreased from 70.5 taps during unimanual tapping to 58.4 taps during bimanual trials (57.8 taps when tapping was combined with circle tracing and 59.1 taps when combined with square tracing). Tap trace length increased from $1.8 \mathrm{~mm}$ during unimanual tapping to $2.4 \mathrm{~mm}$ (being similar for tapping combined with circle as well as square tracing) during bimanual tapping. There were no significant interactions.

Post-hoc comparisons for age group revealed that regarding the number of taps age group 4 tapped significantly more often than the three younger age groups $(p<.001$ for age group 1 and $2 ; p<.05$ for age group 3 ). Additionally, age group 3 tapped significantly more than the children in age group $1(p<.05)$. For tap trace length, a significant difference was found between age group 1 and age group 3 and $4(p<.05$ and $p<.001$, respectively) and between age group 2 and $4(p<.05)$.

The analysis regarding the percentage of intermanual interference for the non-preferred hand due to bimanual performance showed that the age group effect was no longer significant $(F(3,36)=0.66, p=.56$ for number of taps, and $F(3,36)=1.04, p=.39$ for tap trace length) (see Fig. $4 \mathrm{a}$ and b). The effect of shape was also not significant $(F(1,36)=0.02, p=.89$ and $F(1,36)=0.08, p=.77$, for number of taps and tap trace length, respectively), meaning that the percentage of intermanual interference on number and trace length of the taps performed with the non-preferred hand was not significantly different when performing the circle or square with the preferred hand (see Fig. $4 \mathrm{a}$ and b). Children tapped $22.8 \%$ vs. $20.5 \%$ less with an increase in tap trace length of $25.5 \%$ vs. $24.6 \%$ in the bimanual task due to interference of circle and square tracing, respectively.

\subsection{Unimanual vs. bimanual: Tracing}

Because a significant effect of trial was found in the bimanual tracing task $(F(1,36)=$ $5.27, p<.05$ ) with faster tracing during trial 2 , we only compared unimanual tracing with the first bimanual tracing trial. As with tapping, a significant main effect of age group $(F(3,36)=28.67, p<.001)$, condition $(F(1,36)=132.47, p<.001)$, and shape $(F(1,36)=$ $465.73, p<.001)$ were found for preferred hand tracing in the unimanual and bimanual tasks. Older children traced the patterns with a higher velocity than the younger ones, as shown in Fig. 3c. Velocity was considerably slower during bimanual tracing with a mean of $15.7 \mathrm{~cm} / \mathrm{s}$, compared to a mean of $24.5 \mathrm{~cm} / \mathrm{s}$ during unimanual tracing. Furthermore, faster tracing was observed for the circle than for the square. There was a significant interaction of age group and condition $(F(3,36)=5.18, p<.01)$, showing that the participants in the different age groups did not have the same pattern of performance in both conditions. Additionally, a significant interaction between age group and shape was found $(F(3,36)=35.44, p<.001)$. Age group differences were more pronounced when tracing the circle than tracing the square, as can be seen in Fig. 3c. A significant interaction was also observed between condition and shape $(F(1,36)=88.13, p<.001)$. Differences between uniand bimanual tracing speed were much larger when tracing the circle than tracing the square. Finally, a significant three-way interaction was observed between age group, condition and shape $(F(1,36)=3.60, p<.05)$. 

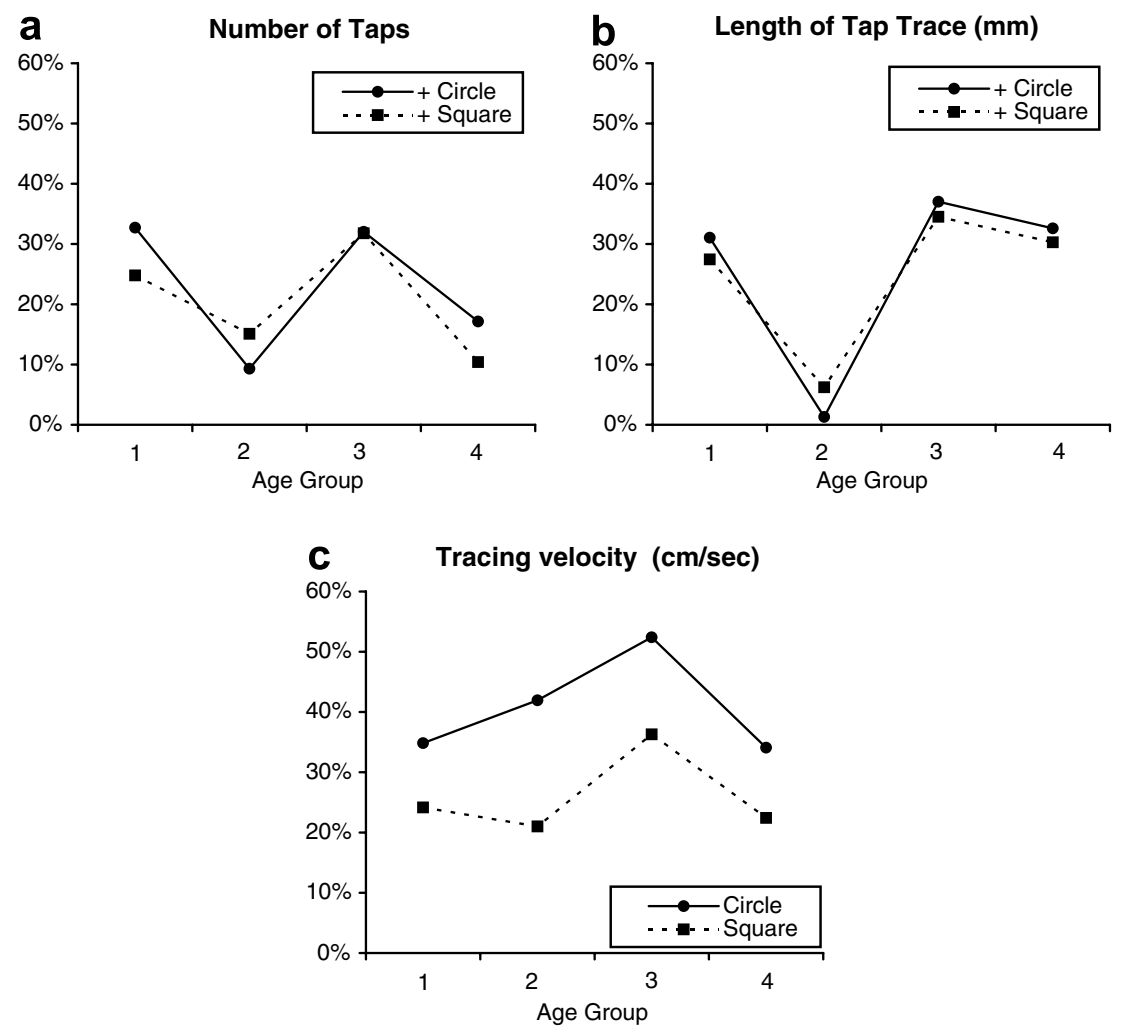

Fig. 4. Effects of age group and shape (circle vs. square) for percentage of intermanual interference for number of taps (a), length of tap trace (b) and mean tracing velocity (c). Note that the percentage of interference for number of taps and length of tap trace are averaged over two trials and are displayed for bimanual trials combined with either circle or square tracing.

Post-hoc tests again revealed that the eldest age group (age group 4) performed superior in comparison to the three younger age groups regarding tracing velocity $(p<.001)$. Age group 3 was significantly faster than age group $1(p<.05)$.

The analysis regarding the percentage of intermanual interference for the preferred hand due to bimanual performance showed that the age group effect was no longer significant $(F(3,36)=1.33, p=.28)$. The effect of shape, however, was highly significant $(F(1,36)=35.48, p<.001)$. The percentage of interference for the preferred hand was much larger when tracing the circle $(41 \%)$ than tracing the square $(26 \%)$, as can be seen in Fig. $4 \mathrm{c}$.

\section{Discussion}

The aim of this study was to examine the development of performance and intermanual interference in a bimanual dual motor task. Children had to tap with their preferred and non-preferred hand and trace a circle or a square with their preferred hand using a pen, either unimanually or bimanually. Unimanual and bimanual performance was compared across four age groups ranging from 4 to 11 years. The results showed that all children tapped more and with shorter tap trace lengths when tapping with the preferred hand, in 
line with results from other studies (Duchêne, Ramackers, Njiokiktjien, \& Vranken, 1991; Njiokiktjien, Sonneville, Kurgansky, \& Vranken, 1997). Furthermore, the data showed that for all age groups performance was better for all variables (number of taps, tap trace length and tracing velocity) in the unimanual condition than in the bimanual condition. This is consistent with results reported by others in studies involving children (Njiokiktjien et al., 1997; Robertson, 2001; Steese-Seda, Brown, \& Caetano, 1995) as well as adults (Annett, 1970; Kelso et al., 1979; Wyke, 1968, 1969, 1971).

Additionally, results showed that older children performed better on both the unimanual and bimanual task than younger children, as evidenced by more taps with shorter tap trace lengths and a higher tracing velocity for the square and circle. An age related improvement in unimanual tasks has been shown in several studies involving tapping (Duchêne et al., 1991; Njiokiktjien et al., 1997) and drawing tasks (Blank, Miller, Von Voss, \& Von Kries, 1999; Blank, Miller, \& Von Voss, 2000; Broderick \& Laszlo, 1987; Van Mier, 2006; Van Mier, Hulstijn, \& Meulenbroek, 1994; Vlachos \& Bonoti, 2004).

Similarly, studies using bimanual tasks also showed an age related performance increase (Fagard \& Marks, 2000; Fagard et al., 2001; Marion et al., 2003). However, in contrast to our expectations the percentage of intermanual interference in our dual task was not significantly different in all age groups. Even though older children performed better on both tasks, the level of interference between the two independent movements (tapping and tracing) was comparable to that seen in younger children.

As anticipated, more interference regarding tracing velocity was found when tapping was combined with tracing the circle than with tracing the square, stressing the importance of the temporal aspects of both movements in bimanual coordination. Research has shown that performing two independent movements simultaneously leads to intermanual interference (Franz et al., 1991; Heuer, 1996; Kelso et al., 1983; Konzem, 1987; Schmidt \& Lee, 2005; Swinnen \& Walter, 1988; Vangheluwe et al., 2004; Walter \& Swinnen, 1990, 1992). More specifically, distinct timing dynamics have been found to be associated with the temporal control of continuous, discontinuous and discrete movements, as in unimanual tapping and drawing (Robertson et al., 1999; Zelaznik, Spencer, \& Doffin, 2000). We found that when both hands had to perform movements using a quite different timing dynamic, i.e., continuous (circle) vs. discrete (tapping) movements, performance of the preferred hand (tracing the shape) slowed down more than when movements had a more similar timing dynamic as in tapping (discrete) and square tracing (discontinuous).

Interference between both hands can be explained by the motor programming theory (Schmidt \& Lee, 2005). This theory states that a single motor program controls each action. Thus, when performing different movements in bimanual actions, two motor programs are simultaneously active, generally leading to interference of one program with the other. In the current study, a clear effect of shape was found with more interference for the circle than the square during bimanual trials. Thus, differences in speed between unimanual and bimanual tracing were more pronounced when tracing the circle, i.e., when timing mechanisms were more different. The latter is in agreement with Schmidt's motor program theory, which states that actions with more similar timing mechanisms, i.e., tapping and tracing the square, are less difficult to combine due to less interference between two similar motor programs (Deutsch, 1983; Kelso et al., 1979; Klapp, 1979, 1981; Peters, 1977; Robertson, 2001; Schmidt \& Lee, 2005). Combining two motor programs with different timing dynamics might require more practice in establishing a third motor program. 
Others have suggested that interference might occur because of a natural tendency to synchronize the upper limbs spatially and temporally and overcoming this tendency to synchronize both movements requires considerable practice (Swinnen, Walter, Lee, \& Serrien, 1993). It might be easier to synchronize tapping and square tracing than tapping and circle tracing because of the timing similarity in the former.

While interference was more pronounced for circle tracing than for square tracing, interference on tapping with the non-preferred hand was not differentially affected by tracing different shapes with the preferred hand. Decreases in the number of taps and increases in tap trace length were not significantly different when the preferred hand traced a circle or a square. Thus, the decrease in speed during circle tracing in the bimanual condition was not caused by an increase in the number of taps or a decrease in tap trace length. It seems that interference was more pronounced for the preferred hand (tracing) then for the nonpreferred hand (tapping). However, this was only in relation to circle tracing. The percentages of interference for tapping and square tracing were around $24 \%$, while interference for circle tracing was $41 \%$. So, only for circle tracing, interference was more pronounced from the non-preferred to the preferred hand. In a study in which participants either performed bimanual tapping or circling tasks, Semjen (2002) found similar timing patterns in both tasks when both hands tapped or circled under isofrequency conditions. Although the findings suggest similar timing mechanisms for tapping and circling, differences appeared under multifrequency conditions, with the latter being comparable to differences in timing in our study.

With respect to interference resulting in deviations in movement paths, we observed that in the bimanual task the tap traces resembled small lines or small curved lines rather than actual dots. Due to the use of cutout patterns for the circle and square, obviously no deviations were found during tracing. We did not find an influence of the non-preferred hand to the preferred hand, in the sense that we observed no pen lifts or (extra) stops during tracing. Since, we did not test the reverse (tapping with the preferred hand and tracing with the non-preferred hand) no additional information on the direction of interference can be given. However, in a follow-up study we asked right-handed children aged 4-10 years to continuously and simultaneously draw a vertical line and a circle with the preferred and non-preferred hands. We replicated our finding regarding more interference for circle drawing than for line drawing. Additionally we found more interference for the preferred hand compared to the non-preferred hand for both circle and line drawing (Smeets \& Van Mier, 2006).

Even though performance increased with age, percentage of interference did not. Children in each age group had the same level of proficiency regarding combining both hand movements. As mentioned in the introduction, the level of bimanual coordination, i.e., inter- and intra-limb coordination has often been linked to the development of the corpus callosum. The majority of interhemispheric transfer is directed through the corpus callosum (Andres et al., 1999; Berlucchi et al., 1995; Cardoso de Oliveira, 2002; Fagard et al., 2001; Jeeves, Silver, \& Milner, 1988; Kennerley et al., 2002; Michel et al., 1985; Ramsey et al., 1979). The major part of the corpus callosum develops after birth; especially the posterior regions 'grow' between 5 and 18 years of age mainly through myelinisation of the fibers, allowing faster and better interhemispheric transmission (Giedd et al., 1996, 1999; Paus et al., 2001). In contrast, the anterior region of the corpus callosum reaches adult size around 4 years of age (Giedd et al., 1996). The involvement of the corpus callosum in bimanual coordination was investigated more specific by Kennerley et al. (2002), who 
showed that callosotomy patients had problems in coordinating bimanual continuous tapping and circle drawing movements but not bimanual discrete tapping movements. The authors propose that in the latter temporal coupling is controlled by subcortical areas, while the temporal-spatial synchronization between the hands in continuous movements involves interhemispheric communication through the corpus callosum. This leads us to believe that interhemispheric transmission might play a more important role in coordinating continuous movements performed by both hands, but less so in the dual task used in our study, combining continuous and discrete movements. Our finding that even the youngest children were able to perform the dual task underscribes this idea. The fact that the level of interference did not show an age-related decrease might also be a consequence of the anterior part of the corpus callosum being already fully developed in all children. While research by Eliassen, Baynes, and Gazzaniga (2000) suggests that the posterior part of the corpus callosum plays a key role in spatial coupling, Preilowski (1972) reported disruption of the temporal coordination between both hands in anterior callosotomy. Our task, in which temporal aspects played a larger role than spatial aspects, might have been more appealing to the anterior part of the corpus callosum, resulting in similar levels of interference in children aged $4-11$ years.

\section{Conclusion}

Little research has been done on the developmental and intermanual interference aspects of bimanual coordination in a dual task. The current study showed that dual task coordination could be observed in 4 year-old children. Age related effects were found in all conditions. Overall, children performed better with the preferred hand and on the unimanual tasks. With respect to bimanual trials, older children tapped more often with shorter tap trace length and they traced the shapes with a higher speed. However, this increase in performance was not related to a decrease in intermanual interference. Throughout all age groups, the level of intermanual interference was more or less similar. Interference was more pronounced when tracing the circle than the square, suggesting that it is more difficult to combine movements with (more) dissimilar timing dynamics. Thus, while children at the age of 4 are able to perform dual tasks, intermanual interference remained the same to at least 11 years of age. We propose that bimanual tasks involving temporal coupling are most likely subserved by subcortical areas or the anterior part of the corpus callosum.

\section{Acknowledgements}

The authors wish to thank the children, their parents, and the teachers of AFNORTH international school, for their willingness to participate and collaborate in this study. Additionally, the authors wish to thank an anonymous reviewer and Jacqueline Fagard for their insightful comments.

\section{References}

Andres, F. G., Mima, T., Schulman, A. E., Dichgans, J., Hallett, M., \& Gerloff, C. (1999). Functional coupling of human cortical sensorimotor areas during bimanual skill acquisition. Brain, 122, 855-870.

Annett, M. (1967). The binomial distribution of right, mixed and left handedness. Quarterly Journal of Experimental Psychology, 19, 327-333. 
Annett, M. (1970). The growth of manual preference and speed. British Journal of Psychology, 61, 545-558.

Armatas, C. A., Summers, J. J., \& Bradshaw, J. L. (1994). Mirror movements in normal adult subjects. Journal of Clinical Experimental Neuropsychology, 16, 405-413.

Berlucchi, G., Aglioti, S., Marzi, C. A., \& Tassinari, G. (1995). Corpus callosum and simple visuomotor integration. Neuropsychologia, 33, 923-936.

Blank, R., Miller, V., Von Voss, H., \& Von Kries, R. (1999). Effects of age on distally and proximally generated drawing movements: A kinematic analysis of school children and adults. Developmental Medicine and Child Neurology, 41, 592-596.

Blank, R., Miller, V., \& Von Voss, H. (2000). Human motor development and hand laterality: A kinematic analysis of drawing movements. Neuroscience Letters, 295, 89-92.

Briggs, G. G., \& Nebes, R. D. (1975). Patterns of hand preference in a student population. Cortex, 11, $230-238$.

Broderick, P., \& Laszlo, J. I. (1987). The drawing of squares and diamonds: A perceptual-motor task analysis. Journal of Experimental Child Psychology, 43, 44-61.

Bruner, J. S. (1970). The growth and structure of skill. In K. Connolly (Ed.), Mechanics of motor skill development. New York: Academic.

Cardoso de Oliveira, S. (2002). The neuronal basis of bimanual coordination: Recent neurophysiological evidence and functional models. Acta Psychologica, 110, 139-159.

Connolly, K., \& Dalgleish, M. (1989). The emergence of a tool-using skill in infancy. Developmental Psychology, 25, 894-912.

De Jong, W. P., Hulstijn, W., Kosterman, B. J. M., \& Smits-Engelsman, B. C. M. (1996). OASIS software and its application in experimental handwriting research. In M. L. Simner, C. G. Leedham, \& A. J. W. M. Thomassen (Eds.), Handwriting and drawing research: Basic and applied issues (pp. 429-440). Amsterdam: IOS Press.

Deutsch, D. (1983). The generation of two isochronous sequences in parallel. Perception and Psychophysics, 34 , 331-337.

Duchêne, R., Ramackers, G., Njiokiktjien, C., \& Vranken, M. (1991). Sensory-motor Development III: Bimanual movements as a timed manoeuvre. In G. Ramaekers \& C. Njiokiktjien (Eds.), Pediatric behavioural neurology (pp. 129-162). Amsterdam: Suyi Publications.

Eliassen, J. C., Baynes, K., \& Gazzaniga, M. S. (2000). Anterior and posterior callosal contributions to simultaneous bimanual movements of the hands and fingers. Brain, 123, 2501-2511.

Fagard, J. (1991). Synchronization and desynchronization in bimanual coordination: A developmental perspective. In J. Fagard \& P. H. Wolff (Eds.), The development of timing control and temporal organization in coordinated action. Amsterdam, The Netherlands: Elsevier Science Publishers.

Fagard, J., \& Jacquet, D. Y. (1989). Onset of bimanual coordination and symmetry versus asymmetry of movement. Infant Behavior and Development, 12, 229-235.

Fagard, J., Hardy-Léger, I., Kervella, C., \& Marks, A. (2001). Changes in interhemispheric transfer rate and the development of bimanual coordination during childhood. Journal of Experimental Child Psychology, 80, $1-22$.

Fagard, J., \& Marks, A. (2000). Unimanual and bimanual tasks and the assessment of handedness in toddlers. Developmental Science, 3, 137-147.

Fowler, B., Duck, T., Mosher, M., \& Mathieson, B. (1991). The coordination of bimanual aiming movements: Evidence for progressive desynchronization. The Quarterly Journal of Experimental Psychology, 43, $205-221$.

Franz, E. A., Zelaznik, H. N., \& McCabe, G. (1991). Evidence of common timing processes in the control of manual, orofacial, and speech movements. Journal of Motor Behavior, 24, 281-287.

Giedd, J. N., Blumenthal, J., Jeffries, N. O., Rajapakse, J. C., Vaituzis, A. C., Liu, H., et al. (1999). Development of the human corpus callosum during childhood and adolescence: A longitudinal MRI study. Progression in Neuropsychopharmacology and Biological Psychiatry, 23, 571-588.

Giedd, J. N., Rumsey, J. M., Castellanos, F. X., Rajapakse, J. C., Kaysen, D., Vaituzis, A. C., et al. (1996). A quantitative MRI study of the corpus callosum in children and adolescents. Developmental Brain Research, 91 , 274-280.

Goldfield, E. C., \& Michel, G. F. (1986a). The ontogeny of infant bimanual reaching during the first year. Infant Behavior and Development, 9, 87-95.

Goldfield, E. C., \& Michel, G. F. (1986b). Spatio-temporal linkage in infant interlimb coordination. Developmental Psychobiology, 19, 259-364.

Heuer, H. (1996). Coordination. In H. Heuer \& S. W. Keele (Eds.), Motor skills (Vol. 2). Handbook of perception and action (pp. 121-180). San Diego: Academic Press.

Ibbotson, N. R., \& Morton, J. (1981). Rhythm and dominance. Cognition, 9, 125-138. 
Jeeves, M. A., Silver, P. H., \& Milner, A. B. (1988). Role of the corpus callosum in the development of a motor skill. Developmental Neuropsychology, 44, 305-323.

Kelso, J. A. S., Southard, D. L., \& Goodman, D. (1979). On the coordination of two-handed movements. Journal of Experimental Psychology: Human Perception and Performance, 5, 229-238.

Kelso, J. A. S., Putnam, C. A., \& Goodman, D. (1983). On the space-time structure of human interlimb co-ordination. Quarterly Journal of Experimental Psychology, 35A, 347-375.

Klapp, S. T. (1979). Doing two things at once: The role of temporal compatibility. Memory and Cognition, 7, 375381.

Klapp, S. T. (1981). Temporal compatibility in dual motor tasks II: Simultaneous articulation and hand movements. Memory and Cognition, 9, 398-401.

Kennerley, S. W., Diedrichsen, J., Hazeltine, E., Semjen, A., \& Ivry, R. B. (2002). Callosotomy patients exhibit temporal uncoupling during continuous bimanual movements. Nature Neuroscience, 5, 376-381.

Kimmerle, M., Mick, L. A., \& Michel, G. F. (1995). Bimanual role-differentiated toy play during infancy. Infant Behavior and Development, 18, 299-307.

Konzem, P. B. (1987). Extended practice and patterns of bimanual interference. Doctoral dissertation, University of Southern California.

Lee, T. D., Swinnen, S. P., \& Verscheuren, S. (1995). Relative phase alterations during bimanual skill acquisition. Journal of Motor Behavior, 27, 263-274.

Marion, S. D., Kilian, S. C., Naramor, T. L., \& Brown, W. S. (2003). Normal development of bimanual coordination: Visuomotor and interhemispheric contributions. Developmental Neuropsychology, 23, 399-421.

Michel, G. F., Ovrut, M. R., \& Harkins, D. A. (1985). Hand-use preference for reaching and object manipulation in 6-13-month-old infants. Genetic, Social and General Psychology Monography, 4, 407-427.

Njiokiktjien, C., Sonneville, L. D., Kurgansky, A. M. H. A., \& Vranken, V. V. M. (1997). Unimanual and bimanual simultaneous fingertapping in schoolchildren: Developmental aspects and hand preference-related asymmetries. Laterality: Asymmetries of Body, Brain, and Cognition, 2, 117-135.

Paus, T., Collins, D. L., Evans, A. C., Leonard, G., Pike, B., \& Zijdenbos, A. (2001). Maturation of white matter in the human brain: A review of magnetic resonance studies. Brain Research Bulletin, 54, 255-266.

Peters, M. (1977). Simultaneous performance of two motor activities: The factor of timing. Neuropsychologica, 15, 461-464.

Peters, M. (1981). Attentional asymmetries during concurrent bimanual performance. Quarterly Journal of Experimental Psychology, 33A, 95-103.

Peters, M. (1985). Constraints in the performance of bimanual tasks and their expression in skilled and unskilled subjects. The Quarterly Journal of Experimental Psychology, 37, 171-196.

Preilowski, B. F. (1972). Possible contribution of the anterior forebrain commissures to bilateral motor coordination. Neuropsychologia, 10, 267-277.

Ramsey, D. S., Campos, J. J., \& Fenson, L. (1979). Onset of bimanual handedness in infants. Infant Behavior and Development, 2, 69-76.

Ramsey, D. S., \& Weber, S. L. (1986). Infants' hand preference in a task involving complementary roles for the two hands. Child Development, 57, 300-307.

Rauch, R. A., \& Jinkins, J. R. (1994). Analysis of cross-sectional area measurements of the corpus callosum adjusted for brain size in male and female subjects from childhood to adulthood. Behavioral Brain Research, 64, 65-78.

Robertson, D. S. (2001). Development of bimanual skill: The search for stable patterns of coordination. Journal of Motor Behavior, 33, 114-126.

Robertson, S. D., Zelaznik, H. N., Lantero, D. A., Bojczyk, K. G., Spencer, R. M., Doffin, J. G., et al. (1999). Correlations for timing consistency among tapping and drawing tasks: Evidence against a single timing process for motor control. Journal of Experimental Psychology Human Perception and Performance, 25, 1316-1330.

Semjen, A. (2002). On the timing basis of bimanual coordination in discrete and continuous tasks. Brain and Cognition, 48, 133-148.

Schmidt, R. A., \& Lee, T. D. (2005). Motor control and learning: A behavioral emphasis (4th ed.). Champaign, IL: Human Kinetics.

Schmidt, R. A., Zalaznik, H. N., Hawkins, B., Frank, J. S., \& Quinn, J. T. (1979). Motor output variability: A theory for the accuracy of rapid motor acts. Psychological Review, 86, 415-451.

Serrien, D. J., Pogosyan, A. H., \& Brown, P. (2004). Cortico-cortical coupling patterns during dual task performance. Experimental Brain Research, 157, 79-84. 
Siegler, S. S., \& Munakata, Y. (1993). Beyond the immaculate transition: Advances in the understanding of change. SRCD Newsletter, January-February, 3-11.

Smeets, D. J., \& Van Mier, H. I. (2006). Developmental changes and intermanual interference in line and circle drawing as an effect of hand dominance. In Fifth Dutch Endo-Neuro-Psycho meeting, 6-9 June, Doorwerth, The Netherlands, pp. 257.

Steese-Seda, D., Brown, S., \& Caetano, C. (1995). Development of visuomotor coordination in school-age children: The bimanual coordination test. Developmental Neuropsychology, 11, 181-199.

Summers, J. J., Todd, J. A., \& Kim, Y. H. (1993). The influence of perceptual and motor factors on bimanual coordination in a polyrhythmic tapping task. Psychological Research, 55, 107-115.

Swinnen, S. P., \& Walter, C. B. (1988). Constraints in coordinating limb movements. In A. M. Colley \& J. R. Beech (Eds.), Cognition and action in skilled behaviour (pp. 127-143). Amsterdam: North Holland.

Swinnen, S. P., \& Walter, C. B. (1991). Toward a movement dynamics perspective on dual-task performance. Human Factors, 33, 367-387.

Swinnen, S. P., Walter, C. B., Lee, T. D., \& Serrien, D. J. (1993). Acquiring bimanual skills: Contrasting forms of information feedback for interlimb decoupling. Journal of Experimental Psychology: Learning, memory and cognition, 19, 1328-1344.

Todor, J. I., \& Smiley, A. L. (1985). Performance differences between the hands: Implications for studying disruption to limb praxis. In E. A. Roy (Ed.), Neuropsychological Studies of apraxia and related disorders (pp. 309344). Amsterdam: North-Holland.

Van Mier, H. I. (2006). Developmental differences in drawing performance of the dominant and non-dominant hand in right handed boys and girls. Human Movement Science, 25, 657-677.

Van Mier, H. I., Hulstijn, W., \& Meulenbroek, R. G. J. (1994). Movement planning in children with motor disorders: Diagnostic implications of pattern complexity and previewing in copying. Developmental Neuropsychology, 10, 231-254.

Vangheluwe, S., Puttemans, V., Wenderoth, N., Van Baelen, M., \& Swinnen, S. P. (2004). Inter- and intralimb transfer of a bimanual task: Generalisability of limb dissociation. Behavioral Brain Research, 154, 535-547.

Vlachos, F., \& Bonoti, F. (2004). Left- and right-handed children's drawing performance: Is there any difference. Laterality, 9, 397-409.

Walter, C. B., \& Swinnen, S. P. (1990). Asymmetric interlimb interference during the performance of a dynamic bimanual task. Brain and Cognition, 14, 185-200.

Walter, C. B., \& Swinnen, S. P. (1992). Adaptive tuning of interlimb attraction to facilitate bimanual decoupling. Journal of Motor Behavior, 24, 95-104.

Wiesendanger, M., Wicki, U., \& Rouiller, E. (1994). Are there unifying structures in the brain responsible for interlimb coordination. In S. P. Swinnen, H. Heuer, J. Massion, \& P. Casaer (Eds.), Interlimb coordination: Neural, dynamical and cognitive constraints (pp. 179-207). San Diego: Academic Press.

Whitall, J. (1989). A developmental study of the interlimb coordination in running and galloping. Journal of Motor Behavior, 21, 409-428.

Wolff, P. H., Gunnoe, C., \& Cohen, C. (1983). Associated movements as a measure of developmental age. Developmental Medicine and Child Neurology, 25, 417-429.

Wyke, M. (1968). The effect of brain lesions in the performance of an arm-hand precision task. Neuropsychologia, $6,125-134$.

Wyke, M. (1969). Influence of direction on the rapidity of bilateral arm movements. Neurospsychologia, 7, 189194.

Wyke, M. (1971). The effects of brain lesions on the performance of bilateral arm movements. Neuropsychologia, 9, 33-42.

Zelaznik, H. N., Spencer, R. M., \& Doffin, J. G. (2000). Temporal precision in tapping and circle drawing movements at preferred rates is not correlated: Further evidence against timing as a general-purpose ability. Journal of Motor Behavior, 32, 193-199. 